\title{
Supplementary material for: Multi-tracer study of gas trapping in an East Antarctic ice core
}

Kévin Fourteau $^{1}$, Patricia Martinerie ${ }^{1}$, Xavier Faïn ${ }^{1}$, Christoph F. Schaller ${ }^{2}$, Rebecca J. Tuckwell ${ }^{3}$, Henning Löwe ${ }^{4}$, Laurent Arnaud ${ }^{1}$, Olivier Magand ${ }^{1}$, Elizabeth R. Thomas ${ }^{3}$, Johannes Freitag ${ }^{2}$, Robert Mulvaney ${ }^{3}$, Martin Schneebeli ${ }^{4}$, and Vladimir Ya. Lipenkov ${ }^{5}$

${ }^{1}$ Univ. Grenoble Alpes, CNRS, IRD, Grenoble INP, IGE, F-38000 Grenoble, France

${ }^{2}$ Alfred Wegener Institute, Helmholtz Centre for Polar and Marine Research, 27568 Bremerhaven, Germany

${ }^{3}$ British Antarctic Survey, Natural Environment Research Council, Cambridge, UK

${ }^{4}$ WSL Institute for Snow and Avalanche Research (SLF), CH-7260 Davos Dorf, Switzerland

${ }^{5}$ Climate and Environmental Research Laboratory, Arctic and Antarctic Research Institute, St. Petersburg, 199397, Russia

Correspondence to: kevin.fourteau@univ-grenoble-alpes.fr or patricia.martinerie@univ-grenoble-alpes.fr

\section{S1 Pycnometry and Tomography}

\section{S1.1 Derivation of the pycnometry equation}

A schematics of the pycnometry system is depicted in Figure S1. When an object, such as a firn sample, is introduced in the first chamber, a part $V_{\mathrm{S}}$ of the volume becomes inaccessible to the gas in the chamber. The physical basis of the pycnometry is

5 the measurement this inaccessible volume, with an ideal gas dilation.

The ideal gas law reads:

$P\left(V_{1}-V_{\mathrm{s}}\right)=N R T$

where $P$ is the pressure in the chamber, $V_{1}$ the volume of the first chamber, $N$ the number of molecule in the chamber, $R$ the ideal gas constant and $T$ the temperature. The second chamber of volume $V_{2}$ is vacuum pumped, and then connected to the first chamber. After the dilation, the ideal gas law becomes:

$P^{\prime}\left(V_{1}+V_{2}-V_{\mathrm{s}}\right)=N R T$

where $P^{\prime}$ is the pressure after dilation. Thus

$P\left(V_{1}-V_{\mathrm{s}}\right)=P^{\prime}\left(V_{1}+V_{2}-V_{\mathrm{s}}\right)$

Re-organizing the equation yields:

$15 V_{\mathrm{s}}=V_{1}-\frac{\mathcal{R}}{1-\mathcal{R}} V_{2}$

where $\mathcal{R}=P^{\prime} / P$. 


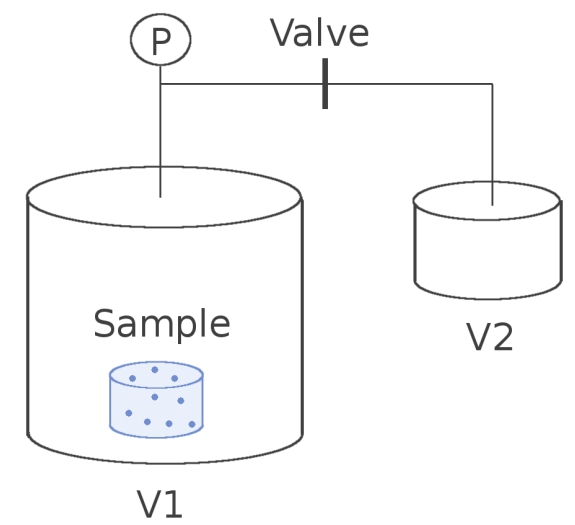

Figure S1. Pycnometry system. The circled $\mathrm{P}$ is the pressure gauge monitoring chamber $V_{1}$

\section{S1.2 Calibration of the chamber volumes}

The usage of the above equations requires the knowledge of the two chamber volumes $V_{1}$ and $V_{2}$. They were estimated by using objects of known volumes. Two types of objects were used, stainless steel balls and bubble free ice cylinders. The stainless steel were bought from Marteau \& Lemarié and respect the ANSI 440C norm. The ball volumes range from to 22 to $46 \mathrm{~cm}^{3}$. The bubble free ice was produced at IGE, and then carved into cylinders using a lathe. Their volume was determined by measuring their mass and knowing the pure density at the recorded cold room temperature. The ice cylinders cover volumes from 19 to $104 \mathrm{~cm}^{3}$. Measurements were also performed without any object, corresponding to the condition $V_{\mathrm{s}}=0 \mathrm{~cm}^{3}$.

In total 24 dilations were performed, using 8 different known volumes $V_{\mathrm{s}}$. The chamber volumes $V_{1}$ and $V_{2}$ were adjusted by a least square method, so the measured volumes $V_{\mathrm{s}}$ match their known values. The measured volumes are displayed Figure $\mathrm{S} 2$ against their known values. The calibration yields $V_{1}=138.96 \pm 0.05 \mathrm{~cm}^{3}$ and $V_{2}=6.875 \pm 0.003 \mathrm{~cm}^{3}$.

\section{S1.3 Uncertainty analysis}

There are seven independent sources of error affecting the estimation of the closed porosity ratio and the density of a cylindrical firn sample: the mass $M$, the radius of the cylinder $r$, the height of the cylinder $h$, the chamber volumes $V_{1}$ and $V_{2}$, and the pressures $P$ and $P^{\prime}$ before and after dilation. The possibility of the sample not to have a perfect cylindrical shape is included in the uncertainties on $r$ and $h$. These errors were quantified during the measurements and propagated to the closed porosity ratio and density using the formula:

$$
(\Delta X)^{2}=\sum_{i}\left(\frac{\partial X}{\partial x_{\mathrm{i}}} \Delta x_{\mathrm{i}}\right)^{2}
$$

where $X$ is either the closed porosity ratio or the density. The $x_{\mathrm{i}}$ correspond to the seven independent sources of error, and $\Delta A$ represents the uncertainty on the variable $A$. The partial derivatives were determined numerically using finite differences. The resulting uncertainties are displayed in Figure S3. It shows that the error on the density of the material is relatively constant 


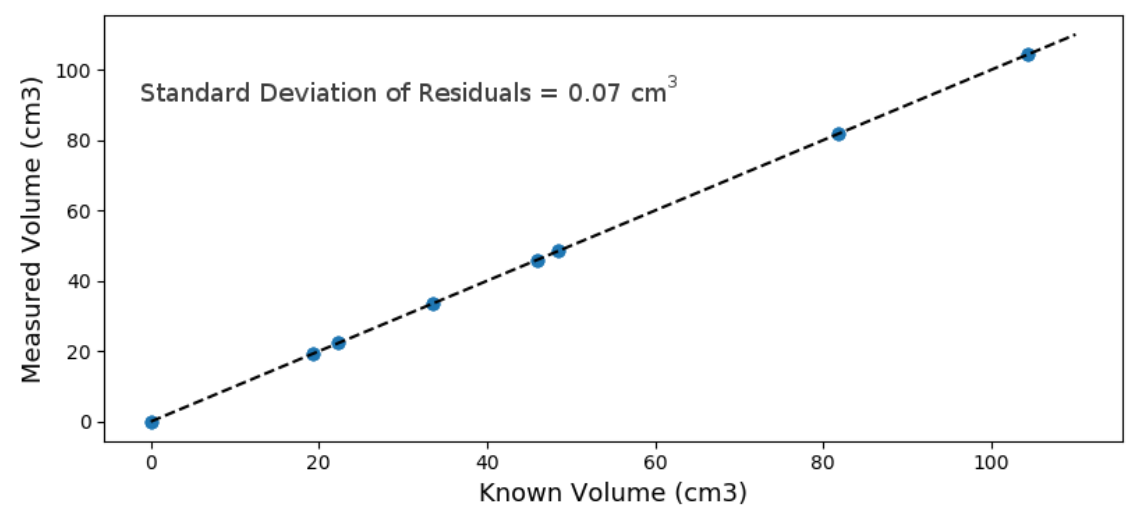

Figure S2. Relationship between the measured volume $V_{\mathrm{s}}$ using pycnometry after calibration, and their known values. The dashed line shows the Known volume = Measured volume line.

and of the order of $0.0082 \mathrm{~g} \mathrm{~cm}^{-3}$, that is to say an uncertainty of 0.09 in relative density to pure ice. On the other hand the closed porosity ratio error grows from 0.02 for low firn densities, up to 0.2 for closed firn.

We also estimated the contribution of each error source. It appears that the error on the radius measurement explains most of the uncertainty on the closed porosity ratio and the density. This is due to the great sensitivity of the volume computation to 5 the value of the radius.
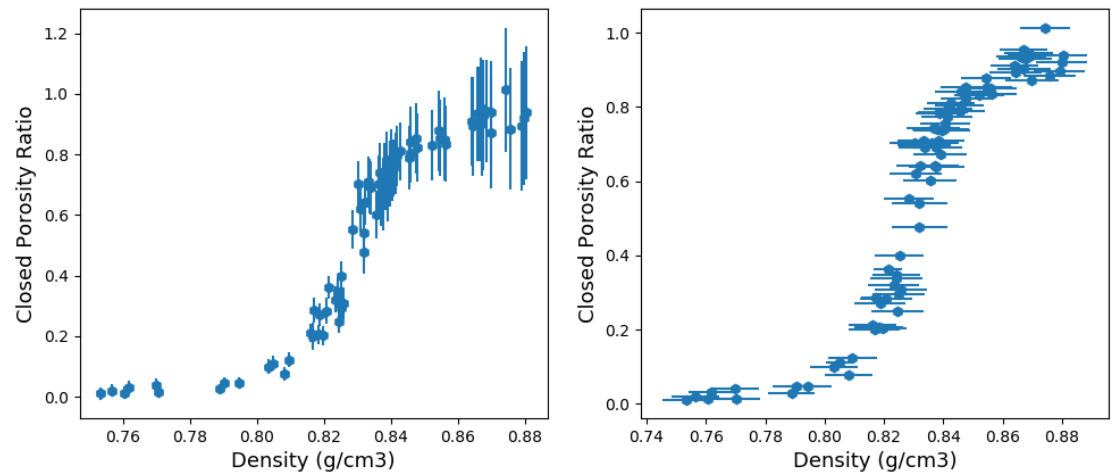

Figure S3. Closed porosity ratio data displayed with uncertainty bars. Left: Uncertainties on the closed porosity ratios. Right: Uncertainties on the densities.

\section{S1.4 Boundary effects on the tomography samples}

Figure S4 shows the variation of relative density and closed porosity ratio along a tomography sample taken at $111.68 \mathrm{~m}$ depth. The vertical dashed lines correspond to the separation between numerical slices. It appears that the first and last $5 \mathrm{~mm}$ of the 
sample are strongly affected by the cut bubbles due the presence of large boundaries at the top and bottom of the samples. These boundary effects manifest as a sharp drop of the closed porosity ratio while approaching the end of the sample. The same behavior is observed for the other tomography samples. Therefore, the first and last slices of each tomography image have been discarded to limit the influence of this large boundary effect.

5 Similarly, in order to estimate the cut-bubble effect, the firn samples were trimmed by an amount of $5 \mathrm{~mm}$ at the top and the bottom and by an amount of $2.5 \mathrm{~mm}$ on the side. This allows to remove the large boundary effect visible on the first few millimeters (visible in Figure S4), while maintaining a final geometry close to the original one.

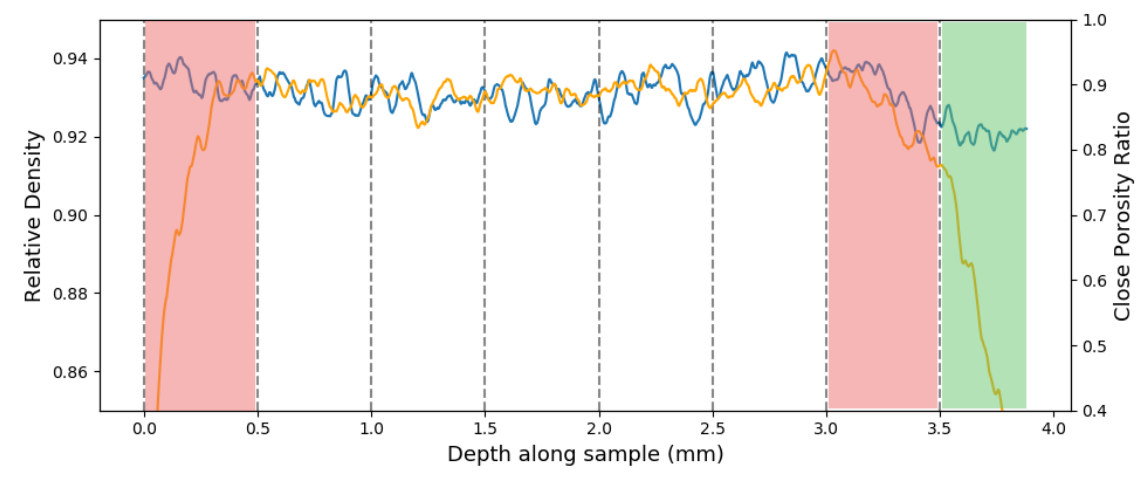

Figure S4. Relative density (blue) and closed porosity ratio (orange) along a tomographic sample taken at $111.68 \mathrm{~m}$ depth. The vertical dashed lines correspond to spacing between the numerical slices. The two red shaded sections are particularly affected by boundary effects. The green section at the end of the sample is less than $5 \mathrm{~mm}$ thick, and is thus not used as a tomographic slice.

\section{S1.5 Impact of cut-bubbles at low densities}

The fraction of re-open porosity due to the cut-bubble effect was estimated in Section 3.1.2 of the main article. For firn samples that are not totally closed, this estimation is based on the tomography images. However, no tomography data is available for relative densities ranging from 0.86 to 0.89 . This implies that the cut-bubble effect is poorly constrained for these densities. In order to estimate a possible impact of this lack of data on the final closed porosity law, we used a second correction relationship, displayed in black in Figure S5. It is expected to overestimate the amount of re-opened bubbles for low values of density. Application of this correction yields the closed porosity ratios depicted in the right panel of Figure S6. For comparison the data obtained using the correction of the main article are displayed on the left panel. The two datasets differ for relative densities below 0.89 , but in a minor fashion. As the closed porosity ratios are low in this part of the curve, the modification of the re-open volume fraction does not result in a large closed porosity ratio change. Therefore, in the case of an underestimation of the re-open volume at low densities, the closed porosity law proposed in the main article is only slightly affected. 


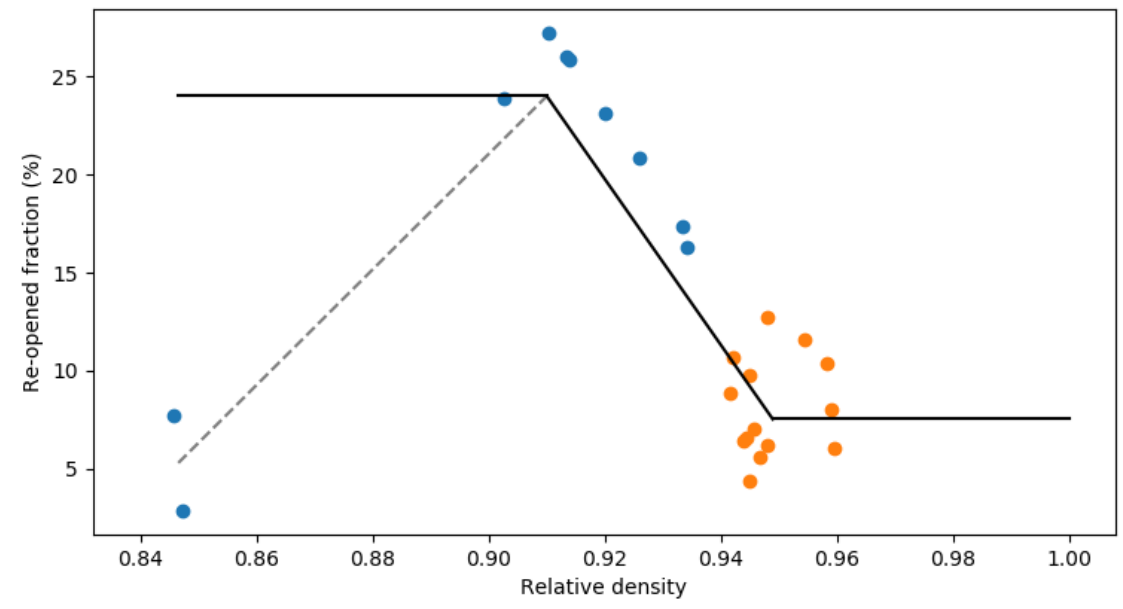

Figure S5. Fraction of re-opened bubbles as a function of relative density. Similarly to the main article, values obtained from tomography samples are represented as blue dots, and values obtained from pycnometry measurements as orange dots. The piecewise linear model used to test the data sensitivity to an overestimation of re-open fraction at low density is shown in black. The dashed line represents the correction used in the main article.
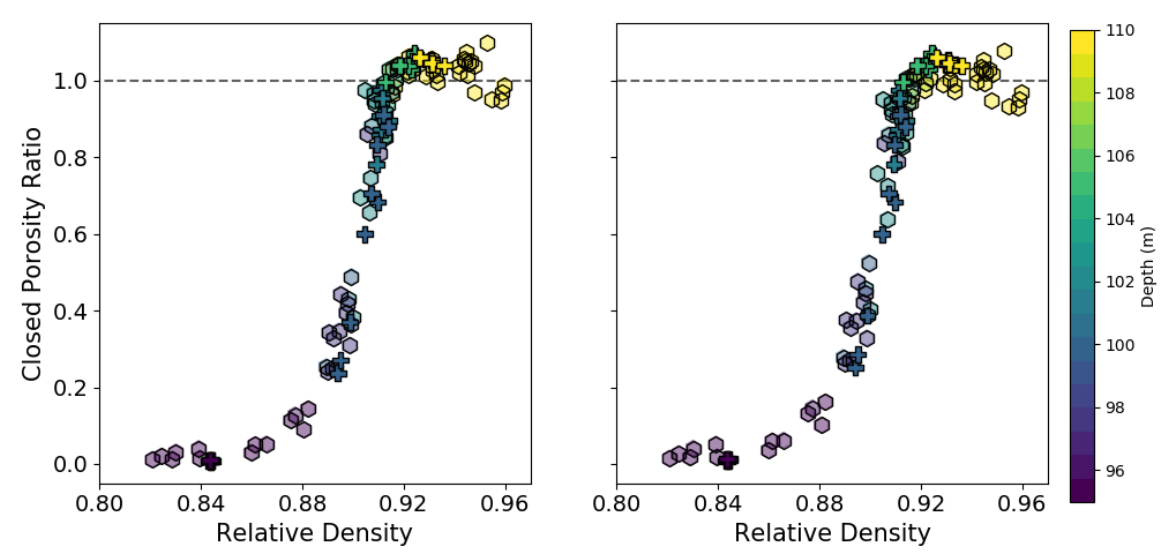

Figure S6. Closed porosity ratios with two different cut-bubble corrections. Left: Using the correction proposed in the main article. Right: Sensitivity test to a large re-open volume fraction at low densities.

\section{S1.6 Uncorrected Vostok pycnometry data}

In the main article Vostok closed porosity data were corrected for the cut-bubble effect using the correction derived for LockIn. However, it is not clear that this correction is suited for the Vostok firn, as it might have a different porous network than Lock-In. For instance Burr et al. (2018) observed a structural difference between the Lock-In and Dome C porous networks. 5 The uncorrected Vostok pycnometry data are displayed in blue Figure S7, alongside with the uncorrected Lock-In data. 


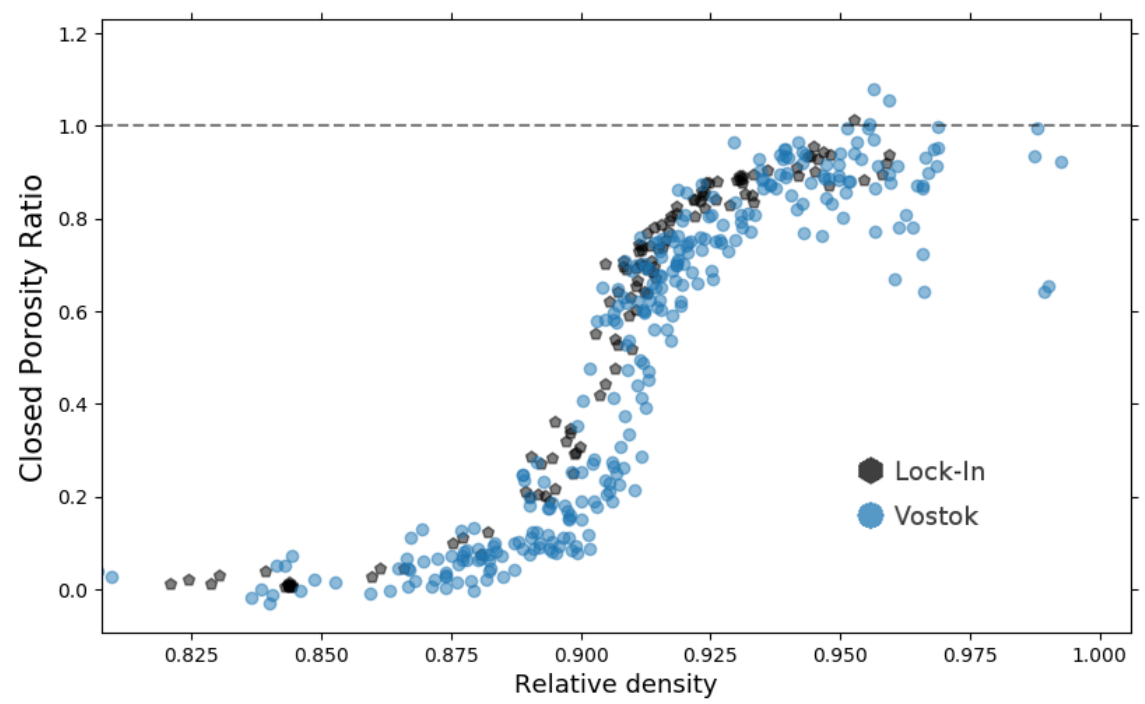

Figure S7. Closed porosity data for Lock-In and Vostok without correction for the cut-bubble effect.

\section{S1.7 Closed porosity versus depth}

The figures provided in the article are plotted using relative density as the abscissa. Here, we display in Figure S8 the closed porosity data as a function of the sample depth. This highlights the firn layering, as samples taken at similar depths display a large variability of closed porosity and density values.

\section{S2 Ion Chromatography}

In total four one-meter long sections were analyzed using ion chromatography. One of the sections is displayed in Figure 8 of the main article. The three remaining sections are displayed in Figure S9 to S11 with density, liquid conductivity and major ion concentrations data. 


\section{Pycnometry}

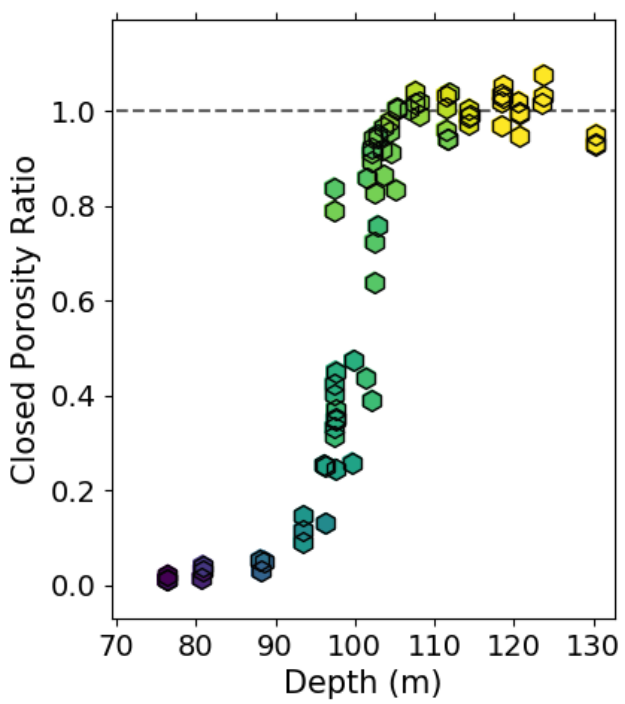

Tomogaphy

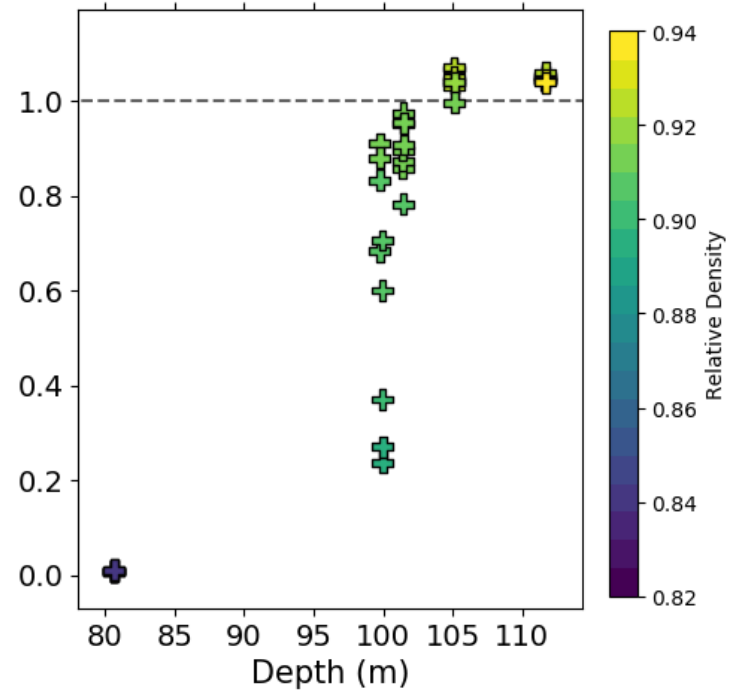

Figure S8. Closed porosity ratios versus sample depth. Colors stand for the measured relative density.

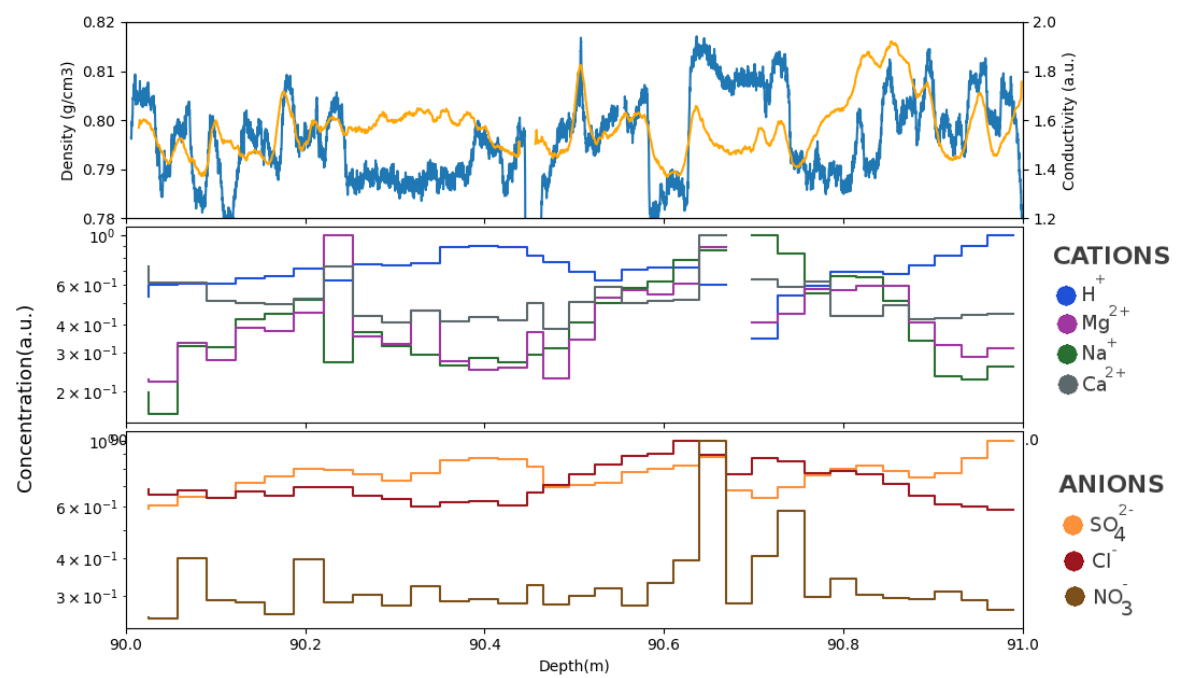

Figure S9. High resolution density (in blue), liquid conductivity (in orange) and major ion concentrations for the section between 90 and $91 \mathrm{~m}$ depth. The ion concentrations are split between anion and cations, and normalized to emphasis variability.

\section{S3 Gas trapping model}

To model the air content in ice, we used a modified version of the Rommelaere et al. (1997) gas trapping model, introducing a limited compressibility for the closed pores. Here, we describe how the new equation representing air trapping is obtained. The 


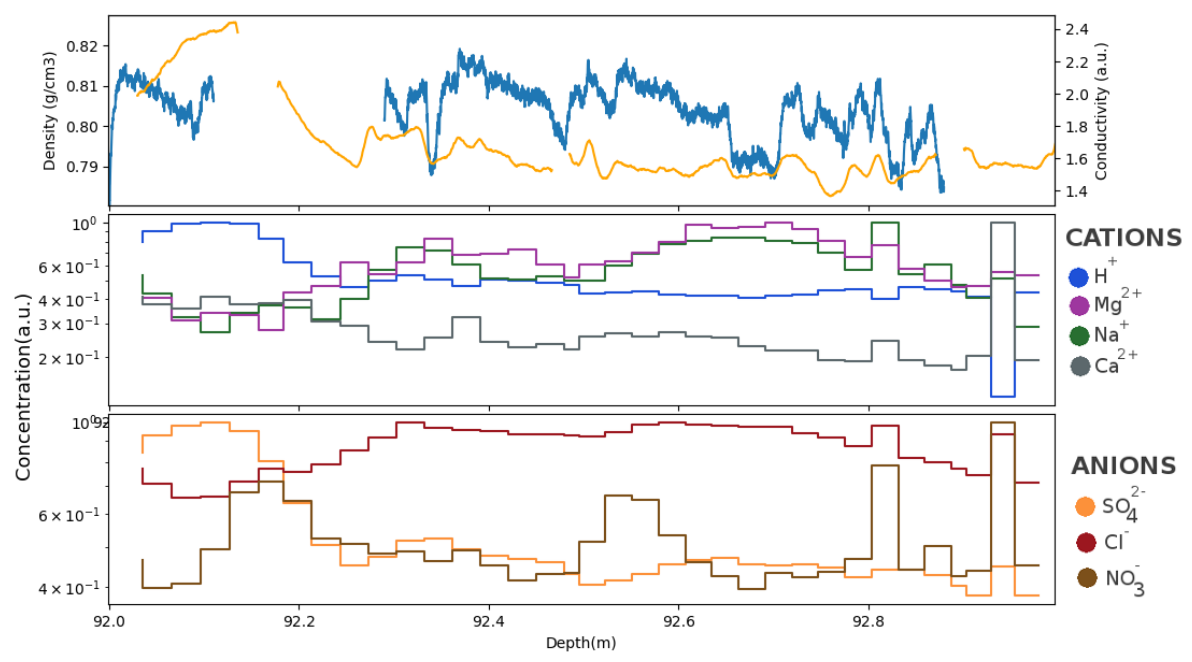

Figure S10. Same as Figure S9 for the section between 92 and $93 \mathrm{~m}$ depth.

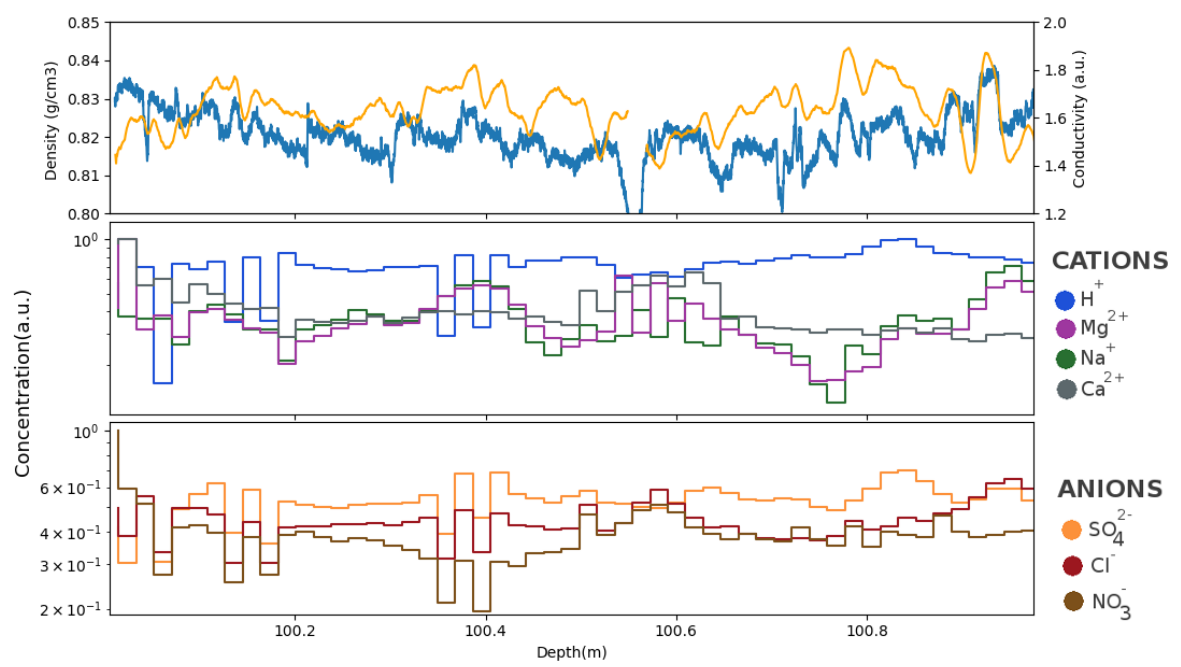

Figure S11. Same as Figure S9 for the section between 100 and $101 \mathrm{~m}$ depth.

derivation is similar to the one of Rommelaere et al. (1997), and we only detail the differences with the original calculation. The symbols used and the quantities they represent are the same as in Rommelaere et al. (1997).

Our new model is based on the replacement of Equation 8 of Rommelaere et al. (1997) by:

$$
\frac{\left(\epsilon_{1}-f_{1}\right)-(\epsilon-f)}{\epsilon-f}=\alpha \frac{\epsilon_{1}-\epsilon}{\epsilon}
$$


where $\epsilon$ and $\epsilon_{1}$ are the total porosity before and after compression, $f$ and $f_{1}$ are the open porosity before and after compression, and $\alpha$ is rate of compression of closed bubbles. This equations simply means that during compression, if the total porosity diminishes by $X \%$, the closed porosity diminishes by $\alpha X \%$. The original study by Rommelaere et al. (1997) corresponds to the case $\alpha=1$.

5

The air conservation in bubbles is expressed by the Equation 13 of Rommelaere et al. (1997). Re-arranging the equations to eliminate the intermediate subscripts 1 , and taking the time step $d t$ as infinitely small leads to the equation:

$d\left[(\epsilon-f) c_{\mathrm{air}}^{\mathrm{b}}\right]=-\partial_{z} v d t(\epsilon-f) c_{\mathrm{air}}^{\mathrm{b}}+\left(d \epsilon\left(1-\alpha+\alpha \frac{f}{\epsilon}\right)-d f\right) c_{\mathrm{air}}$

This equation replaces Equation 15 of Rommelaere et al. (1997).

10 Finally under the assumption of stationarity, this Lagrangian description is converted into an Eulerian one:

$\partial_{z}\left(v q_{\text {air }}^{\mathrm{b}}\right)=-c_{\mathrm{air}} v\left(\partial_{z} f+\partial_{z} \epsilon\left(\alpha-1-\alpha \frac{f}{\epsilon}\right)\right)$

The quantity of air trapped in ice $q_{\mathrm{air}}^{\mathrm{b}}$ can then be calculated from this equation. The required inputs for the model are density and closed porosity profiles, to compute the $v, f$ and $\epsilon$ variables. For this study Equation 8 was solved using a finite difference scheme. 


\section{References}

Burr, A., Ballot, C., Lhuissier, P., Martinerie, P., Martin, C. L., and Philip, A.: Pore morphology of polar firn around closure revealed by X-ray tomography, Cryosphere, 12, 2481-2500, doi:10.5194/tc-12-2481-2018, 2018.

Rommelaere, V., Arnaud, L., and Barnola, J.-M.: Reconstructing recent atmospheric trace gas concentrations from polar firn and bubbly ice data by inverse methods, J. Geophys. Res. Atmos., 102, 30 069-30 083, doi:10.1029/97jd02653, 1997. 